\section{Rectovesical fistula treated by glue injection plus endoclipping technique}

A 74-year-old man was referred to our de-
partment for endoscopic evaluation and
treatment of a rectovesical fistula. He had
undergone prostatectomy for early pros-
tate cancer 2 weeks previously. Post-
operatively, he had developed pneuma-
turia and dysuria; computed tomography (CT) had revealed a rectovesical fistula.

At colonoscopy, a well-circumscribed opening of a fistula tract was seen in the anterior rectal wall, located $2 \mathrm{~cm}$ beyond the linea dentata ( $\boldsymbol{O}$ Fig. $\mathbf{1}$ ). The edges of the fistula were cauterized with argon plasma coagulation to stimulate an inflammatory reaction and local collagen synthesis. A 2-mL syringe containing $1 \mathrm{~mL}$ n-2-butyl cyanoacrylate (Histoacryl; B. Braun, Melsungen, Germany) and $1 \mathrm{~mL}$ Lipiodol was prepared and the glue/Lipiodol mix was injected via a $23-G$ variceal needle in aliquots of $0.5 \mathrm{~mL}$ at four sites of the wall of the fistula, followed by a flush of Lipiodol equivalent in volume to the dead space of the needle. The margins of the fistula orifice were brought into contact and closed with endoclips (HX-600-900; Olympus, Athens, Greece; $\bullet$ Fig. 2). After endoscopic intervention, the patient was treated with parenteral nutrition and antibiotics. His symptoms completely resolved and he was discharged 7 days after the procedure. A follow-up endoscopy performed 1 month later revealed that the lesion was well healed $(\bullet$ Fig. 3 ). He remains asymptomatic 6 months post-procedure. Rectal injury after radical prostatectomy with subsequent formation of rectovesical fistula has an incidence of $1-11 \%$ [1]. York-Mason, modified York-Mason and less invasive repair methods, which include laparoscopic and robotic-assisted techniques, have been reported as successful treatment modalities for rectovesical fistulas $[2,3]$.

We wish to emphasize the use of the combination of endoclips plus n-2-butyl cyanoacrylate injection in the treatment of gastrointestinal fistulas. To our knowledge, the present case is the first report of a rectovesical fistula that was success-

(c) Georg Thieme Verlag KG fully treated with injection of glue plus endoclipping.

Endoscopy_UCTN_Code_TTT_1AQ_2AG

Competing interests: None

\section{P. Katsinelos ${ }^{1}$, S. Gkagkalis ${ }^{1}$, G. Chatzi- mavroudis', C. Zavos ${ }^{2}$, J. Kountouras ${ }^{2}$}

${ }^{1}$ Department of Endoscopy and Motility Unit, G. Gennimatas General Hospital, Thessaloniki, Greece

${ }^{2}$ Second Department of Internal Medicine, School of Medicine, Aristotle University of Thessaloniki, Ippokration Hospital,

Thessaloniki, Greece

\section{References}

1 Harpster LE, Rommel FM, Sieber PR et al. The incidence and management of rectal injury associated with radical prostatectomy in a community urology practice. J Urol 1995; 154: $1435-1438$

2 Kasraeian A, Rozet $F$, Cathelineau $X$ et al. Modified York-Mason technique for repair of iatrogenic rectourinary fistula: the Montsouris experience. J Urol 2009; 181: 1178 1183

3 Sotelo $R$, de Andrade $R$, Carmona $O$ et al. Robotic repair of rectovesical fistula resulting from open radical prostatectomy. Urology 2008; 72: $1344-1346$

\section{Bibliography}

Dol http://dx.doi.org/

10.1055/s-0032-1308945

Endoscopy 2013; 45: E238

Stuttgart · New York

ISSN 0013-726X

\section{Corresponding author}

\section{P. Katsinelos, MD, PhD}

Department of Endoscopy and Motility Unit

G. Gennimatas General Hospital

School of Medicine, Aristotle University of

Thessaloniki

Thessaloniki

Greece

Fax: +30-2310-963341

gchatzimav@yahoo.gr



Fig. 1 Endoscopic view of the orifice of a fistula located in the anterior rectal wall $2 \mathrm{~cm}$ from the linea dentata.

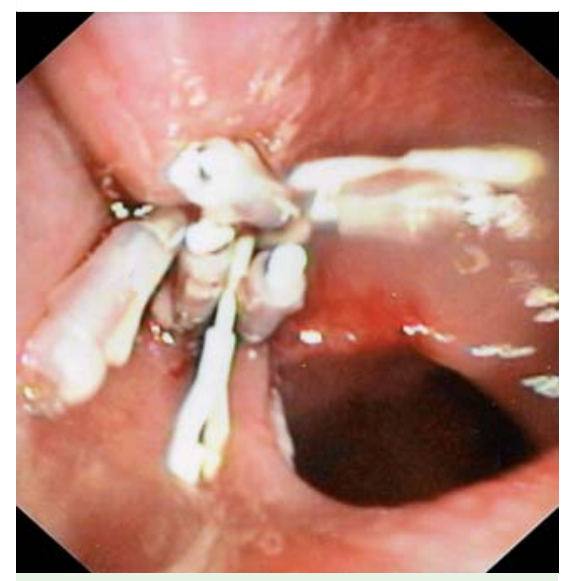

Fig.2 View during the endoscopic procedure to close the fistula using a glue injection plus endoclipping showing complete closure of the orifice of the fistula with the use of six endoclips.

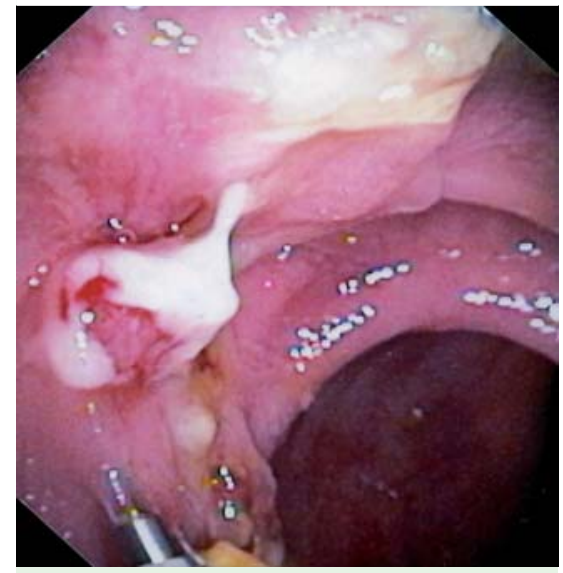

Fig.3 View during an endoscopy performed 1 month later showing that the fistula is well healed and that some residual clips remain in place. 